(C) 2000 Cambridge University Press

\title{
Spatial absorption of a magnetosonic wave in a dusty magnetized plasma
}

\author{
M. C. de JULI and R. S. SCHNEIDER \\ Instituto de Física, Universidade Federal do Rio Grande do Sul, Caixa Postal 15051, \\ 91501-970 Porto Alegre, RS, Brasil (juli@if.ufrgs.br)
}

(Received 20 July 1999 and in revised form 28 January 2000)

\begin{abstract}
The dielectric tensor for a multicomponent magnetized dusty plasma, including the effect of capture of plasma electrons and ions by the dust particles, is rewritten in order to provide expressions more suitable for applications. We use this tensor to study the spatial absorption of a magnetosonic wave, including effects up to second order in the Larmor radius. We analyse the absorption of the wave due to the presence of dust particles with variable charge and the modification of this absorption due to finite-Larmorradius effects.
\end{abstract}

\section{Introduction}

In a previous paper (de Juli and Schneider 1998, hereinafter referred to as paper I), we developed a kinetic theory of a dusty magnetized plasma with variable charge on the dust particles. The dielectric tensor was written as the sum of two terms. One term has components $\tilde{\epsilon}_{i j}^{h}$, and is formally identical with the dielectric tensor of a homogeneous magnetized plasma, with the resonant denominator modified by the addition of a purely imaginary term containing a frequency $v_{\beta d}^{0}$ that characterizes the collisions between plasma particles and dust particles. The other term, with components $\epsilon_{i j}^{N}$, is entirely dependent on the model used to describe the charging process of the dust particles.

In this paper, we rewrite the components of this dielectric tensor in a new form, using a convenient expansion of the Bessel functions, in order to deal more easily with the different powers of the Larmor radius, which are essential for the analysis of the effects of charge variation of the dust particles that are included in $\epsilon_{i j}^{N}$.

We use this tensor to study the propagation of a magnetosonic wave in a dusty magnetized plasma with the variation of the charge of the dust taken into account, and to analyse how the spatial propagation of this wave is influenced by the presence of the dust in the plasma and by the change in the dust charge. We show that spatial absorption occurs owing to the charge variation of the dust particles. This absorption is not significantly changed by contributions connected with finite-Larmor-radius effects included in $\epsilon_{i j}^{N}$.

\section{The dielectric tensor}

In paper I, we obtained the components of the dielectric tensor for a homogeneous dusty magnetized plasma. We consider again a homogeneous plasma composed of particles of charge $q_{\beta}$ and mass $m_{\beta}$, where the subscript 
$\beta=e, i$ identifies electrons and ions respectively, in a homogeneous magnetic field $\mathbf{B}_{0}=B_{0} \mathrm{e}_{z}$. In this magnetized plasma, we consider embedded spherical dust grains with radius $a$ and variable charge $q$; the charge variation originates from collisions between the dust particles and particles of species $\beta$. The assumption of spherical dust grains is valid in the limit $a \ll \lambda_{D} \ll \lambda_{\text {mfp }}$, where $\lambda_{D}$ is the plasma Debye length and $\lambda_{\mathrm{mfp}}$ is the mean free path of electrons and ions.

We are considering Larmor-radius effects arising from the motion of electrons and ions and neglecting this kind of effect for the dust particles, since they are assumed to be immobile because of their large mass. This assumption implies that the regime in which our work is valid is such that $\omega \gg \Omega_{d}$, where $\Omega_{d}$ is the cyclotron frequency of the dust particles.

At the same time, we neglect the influence of the external magnetic field in the charging process of the dust particles, using a cross-section for this process derived from the OML theory (Tsytovich 1997). This is based on a numerical calculation by Chang and Spariosu (1993), which shows that the characteristics of the dust-particles charging process are not significantly influenced by the presence of an external magnetic field if the dust-particle radius $a<\rho_{G}$, where $\rho_{G}$ is the mean gyroradius of the electrons, given by $\rho_{G}=\left(\frac{1}{2} \pi\right)^{1 / 2} r_{L e}$, with $r_{\mathrm{Le}}$ the electron Larmor radius. For the parameter values used in this work, the relation $a<\rho_{G}$ is always satisfied.

The components of the dielectric tensor are written as $\epsilon_{i j}=\tilde{\epsilon}_{i j}^{h}+\epsilon_{i j}^{N}$. The first term is given by

with

$$
\begin{aligned}
\tilde{\epsilon}_{i j}^{h}= & \delta_{i j}+\hat{\epsilon}_{i j}^{h}-\delta_{i z} \delta_{j z} \sum_{\beta} \frac{X_{\beta}}{n_{\beta 0}} \int d^{3} u \frac{u_{\|}}{u_{\perp}} L\left(F_{\beta 0}\right) \\
& +\delta_{j z} \sum_{\beta} \frac{X_{\beta}}{n_{\beta 0}} \sum_{n=-\infty}^{\infty} \int d^{3} u\left[i \frac{\nu_{\beta d}^{0}(u)}{\omega} \frac{L\left(F_{\beta 0}\right)}{D_{n \beta}}\left(\frac{u_{\|}}{u_{\perp}}\right)^{\delta_{i z}}\right] R_{i j}^{n \beta},
\end{aligned}
$$

$$
\hat{\epsilon}_{i j}^{h}=\sum_{\beta} \frac{X_{\beta}}{n_{\beta 0}} \sum_{n=-\infty}^{\infty} \int d^{3} u u_{\perp} \frac{\mathscr{L}\left(F_{\beta 0}\right)}{D_{n \beta}}\left(\frac{u_{\|}}{u_{\perp}}\right)^{\delta_{i z}+\delta_{j z}} R_{i j}^{n \beta} .
$$

In these expressions, $\mathbf{u}=\mathbf{p} / m_{\beta} c, X_{\beta}=\omega_{p \beta}^{2} / \omega^{2}, \omega_{p \beta}^{2}=4 \pi n_{\beta 0} q_{\beta}^{2} / m_{\beta}$ is the square of the plasma frequency of particles of species $\beta, n_{\beta 0}$ and $F_{\beta 0}$ are the equilibrium density and the equilibrium distribution function respectively of particles of species $\beta$,

$$
\mathscr{L}\left(F_{\beta 0}\right)=\frac{1}{\gamma_{\beta}}\left[\left(\gamma_{\beta}-N_{\|} u_{\|}\right) \frac{\partial F_{\beta 0}}{\partial u_{\perp}}+N_{\|} u_{\perp} \frac{\partial F_{\beta 0}}{\partial u_{\|}}\right],
$$

with $N_{\|}=k_{\|} c / \omega, \gamma_{\beta}$ being the relativistic factor, and

Also,

$$
L\left(F_{\beta 0}\right)=\frac{1}{\gamma_{\beta}}\left(u_{\|} \frac{\partial F_{\beta 0}}{\partial u_{\perp}}-u_{\perp} \frac{\partial F_{\beta 0}}{\partial u_{\|}}\right) .
$$

$$
D_{n \beta}=\gamma_{\beta}\left(1+i \frac{\nu_{\beta d}^{0}}{\omega}\right)-N_{\|} u_{\|}-n Y_{\beta} \text {, }
$$

where

$$
v_{\beta d}^{0}=\pi a^{2} n_{d 0} c \frac{u^{2}+\tilde{C}_{\beta}}{u} H\left(u^{2}+\tilde{C}_{\beta}\right), \quad \tilde{C}_{\beta} \equiv \frac{2 Z_{d} e q_{\beta}}{a m_{\beta} c^{2}},
$$

$-Z_{d} e$ being the equilibrium charge of the dust particle and $H$ the Heaviside function, and $Y_{\beta}=\Omega_{\beta} / \omega$ where $\Omega_{\beta}=q_{\beta} B_{0} / m_{\beta} c$ is the cyclotron frequency of 
particles of species $\beta$. The $R_{i j}^{n \beta}$ are combinations of Bessel functions with argument $b_{\beta}=N_{\perp} u_{\perp} / Y_{\beta}$, where $N_{\perp}=k_{\perp} c / \omega$, and their derivatives. Full expressions for the $R_{i j}^{n \beta}$ are given in Appendix A.

The second term of the tensor is given by

where

$$
\epsilon_{i j}^{N}=-\frac{4 \pi i}{\omega} \frac{c^{2}}{\omega^{2}} \frac{1}{1+i\left(\frac{\nu_{d}^{0}}{\omega}+\frac{\nu_{1}}{\omega}\right)} \hat{U}_{i} \hat{S}_{j},
$$

and

$$
\hat{U}_{i}=\sum_{\beta} q_{\beta} \sum_{n=-\infty}^{\infty} \int d^{3} u u_{\perp} \frac{u \sigma^{\prime}(u) F_{\beta 0}}{g_{\beta}-n Y_{\beta}}\left(\frac{u_{\|}}{u_{\perp}}\right)^{\delta_{i z}} R_{i z}^{n \beta}
$$

$$
\begin{aligned}
\hat{S}_{j}= & \sum_{\beta} \frac{q_{\beta}^{2}}{m_{\beta} c} \sum_{n=-\infty}^{\infty} \int d^{3} u \frac{\left[i v_{\beta d}^{0}(u) / \omega\right] \mathscr{L}\left(F_{\beta 0}\right)}{g_{\beta}-n Y_{\beta}}\left(\frac{u_{\|}}{u_{\perp}}\right)^{\delta_{j z}} R_{z j}^{n \beta} \\
& -\delta_{j z} \sum_{\beta} \frac{q_{\beta}^{2}}{m_{\beta} c} \int d^{3} u\left[i \frac{v_{\beta d}^{0}(u)}{\omega} \frac{L\left(F_{\beta 0}\right)}{u_{\perp}}\right] \\
& +\delta_{j z} \sum_{\beta} \frac{q_{\beta}^{2}}{m_{\beta} c} \sum_{n=-\infty}^{\infty} \int d^{3} u \frac{\left[i v_{\beta d}^{0}(u) / \omega\right]^{2}}{g_{\beta}-n Y_{\beta}} \frac{L\left(F_{\beta 0}\right)}{u_{\perp}} R_{z j}^{n \beta} .
\end{aligned}
$$

In these expressions,

$$
\nu_{d}^{0}=-c \sum_{\beta} q_{\beta} \int d^{3} u \sigma_{\beta}^{\prime} u F_{\beta 0},
$$

where $\left.\sigma_{\beta}^{\prime}(u) \equiv\left(\partial \sigma_{\beta} / \partial q\right)\right|_{q=-z_{d} e}$, and the charging cross-section is given (Spitzer 1978) by

$$
\sigma_{\beta}(q, u)=\pi a^{2}\left(1-\frac{q}{Z_{d} e} \frac{\tilde{C}_{\beta}}{u^{2}}\right) H\left(1-\frac{q}{Z_{d} e} \frac{\tilde{C}_{\beta}}{u^{2}}\right) .
$$

Also,

where

$$
\nu_{1}=i c \sum_{\beta} q_{\beta} \sum_{n=-\infty}^{\infty} \int d^{3} u \frac{\nu_{\beta d}^{0}}{\omega} \gamma_{\beta} u \sigma_{\beta}^{\prime} F_{\beta 0} \frac{J_{n}^{2}\left(b_{\beta}\right)}{g_{\beta}-n Y_{\beta}},
$$

$$
g_{\beta} \equiv \gamma_{\beta}\left(1+i \frac{\nu_{\beta d}^{0}}{\omega}\right)-N_{\|} u_{\|} .
$$

This form of the dielectric tensor is very general, under the assumptions that we have made, and was used in paper I to study the propagation of a magnetosonic wave perpendicularly to the external magnetic field. In doing this, in order to evaluate the integrals in the $u$ variable, we make an expansion of the denominator that occurs in them, and which is very common in the literature (Vladimirov 1994; Prasad 1998). This kind of expansion is good if the involved frequencies are sufficiently high that $\omega \sim \omega_{p e} \gg \max \left(k v_{T e}, v_{d}^{0}, v_{\beta d}^{0}\right)$ (Vladimirov 1994), and has as consequence that only a few terms need be retained. If the frequency is lowered, we must retain more and more terms in the expansion, which turns out to be algebraically very expensive. A better approach is to rewrite the general dielectric tensor in a form that is more convenient for regimes with lower frequencies and that at the same time takes care of powers of the Larmor radius in an easy way. 
In order to rewrite this tensor in a more suitable form for application, in all these expressions the summation over $n$ from minus to plus infinity is transformed into a summation running from zero to infinity, and the combinations of Bessel functions and their derivatives are expanded in the following way (Abramowitz and Stegun 1970):

$$
\begin{gathered}
J_{n}^{2}\left(b_{\beta}\right)=\sum_{m=0}^{\infty} a(n, m)\left(b_{\beta}^{2}\right)^{n+m}, \\
J_{n}\left(b_{\beta}\right) J_{n}^{\prime}\left(b_{\beta}\right)=\sum_{m=0}^{\infty}(n+m) a(n, m) b_{\beta}\left(b_{\beta}^{2}\right)^{n+m-1}, \\
J_{n}^{\prime 2}\left(b_{\beta}\right)=\sum_{m=0}^{\infty} d(n, m)\left(b_{\beta}^{2}\right)^{n+m-1},
\end{gathered}
$$

where

$$
a(n, m)=\frac{(-1)^{m}[2(n+m)] !\left(\frac{1}{2}\right)^{2(n+m)}}{[(n+m) !]^{2}(2 n+m) ! m !}
$$

and $d(n, m)=a(1, m-2)$ for $n=0$, and

$$
d(n, m)=\frac{1}{4}\left[a(n-1, m)+a(n+1, m-2)-2 \frac{n+m-1}{n+m} a(n, m-1)\right]
$$

for $n>0$, with the convention $1 /(-m) !=0$.

Then all the components of the dielectric tensor for the wavevector $\mathbf{k}$ in the $(x, z)$ plane can be written in terms of integrals of the form

$$
\tilde{I}_{\beta}(n, m, h, s ; G) \equiv \frac{1}{n_{\beta 0}} \int d^{3} u \frac{u_{\|}^{h} u_{\perp}^{2(n+m-1)} u_{\perp} G}{g_{\beta}+s n Y_{\beta}} .
$$

Using (2.12)-(2.14), the components $\tilde{\epsilon}_{i j}^{h}$ and $\epsilon_{i j}^{N}$ of the dielectric tensor can be written as series in powers of $N_{\perp} / Y_{\beta}$, where in each term we have an integral of the type (2.17). The full expressions for $\tilde{\epsilon}_{i j}^{h}$ and $\epsilon_{i j}^{N}$ are given in Appendix B.

\section{The magnetosonic wave}

The dispersion relation for the magnetosonic wave, which propages perpendicularly to the external magnetic field $\left(N_{\|}=0\right)$, can be written formally as

$$
\Lambda \equiv N^{2}-\frac{\epsilon_{x x} \epsilon_{y y}-\epsilon_{x y} \epsilon_{y x}}{\epsilon_{x x}}=0
$$

We shall use for the equilibrium distribution functions of electrons and ions, Maxwellian distribution functions

$$
F_{\beta 0}=\frac{\mu_{\beta}^{3 / 2} n_{\beta 0}}{(2 \pi)^{3 / 2}} e^{-\mu_{\beta} u^{2} / 2},
$$


where $\mu_{\beta}=m_{\beta} c^{2} / T_{\beta}, T_{\beta}$ being the temperature of particles of species $\beta$, with $T_{e}=T_{i}=T$. The following approximations are made in the calculation of the components of interest of the dielectric tensor presented in Sect. 2 : relativistic effects are neglected, which means that $\gamma_{\beta} \approx 1$; in each component of the dielectric tensor, we retain only contributions up to order $N^{2}$, which implies retaining only the lowest-order contributions in the finite Larmor radius. We obtain all the necessary components of the dielectric tensor in terms of the integrals

$$
\begin{gathered}
i_{n} \equiv \int_{0}^{\sqrt{-\tilde{C}_{e}}} d u u^{n} e^{-\mu_{e} u^{2} / 2}, \\
I(p, n) \equiv \int_{0}^{\infty} d u \frac{u^{n} e^{-\mu_{i} u^{2} / 2}}{\left(1+p Y_{i}\right)^{2} u^{2}+A^{2}\left(u^{2}+\tilde{C}_{i}\right)^{2}}, \\
E(p, n) \equiv \int_{\sqrt{-\tilde{C}_{e}}}^{\infty} d u \frac{u^{n} e^{-\mu_{e} u^{2} / 2}}{\left(1+p Y_{e}\right)^{2} u^{2}+A^{2}\left(u^{2}+\tilde{C}_{e}\right)^{2}},
\end{gathered}
$$

where $A \equiv \pi a^{2} n_{d 0} c / \omega$. The dielectric tensor components of interest for the dispersion relation under consideration, written as $\epsilon_{i j}=\tilde{\epsilon}_{i j}^{h}+\epsilon_{i j}^{N}$, will be rewritten in such a way as to make clear the dependence on $N^{2}$. The terms $\tilde{\epsilon}_{i j}^{h}$ are given by

$$
\begin{gathered}
\tilde{\epsilon}_{x x}^{h}=\left(1+\chi_{x x}^{0 R}+N^{2} \chi_{x x}^{1 R}\right)+i\left(\chi_{x x}^{0 I}+N^{2} \chi_{x x}^{1 I}\right), \\
\tilde{\epsilon}_{y y}^{h}=\tilde{\epsilon}_{x x}^{h}+N^{2}\left(\chi_{y y}^{1 R}+i \chi_{y y}^{1 I}\right), \\
\tilde{\epsilon}_{x y}^{h}=\left(\chi_{x y}^{0 R}+N^{2} \chi_{x y}^{1 R}\right)+i\left(\chi_{x y}^{0 I}+N^{2} \chi_{x y}^{1 I}\right),
\end{gathered}
$$

and $\tilde{\epsilon}_{y x}^{h}=\tilde{\epsilon}_{x y}^{h *}$, where the expressions for the $\chi_{i j}^{0 R}$ and $\chi_{i j}^{0 I}$ are given in Appendix C.

The components $\epsilon_{i j}^{N}$ can be written as

$$
\begin{gathered}
\epsilon_{x x}^{N}=N^{2} C\left(\chi_{x x}^{N R}+i \chi_{x x}^{N I}\right), \\
\epsilon_{y y}^{N}=-N^{2} C\left(\chi_{y y}^{N R}+i \chi_{y y}^{N I}\right), \\
\epsilon_{x y}^{N}=-N^{2} C\left(\chi_{x y}^{N R}+i \chi_{x y}^{N I}\right), \\
\epsilon_{y x}^{N}=-N^{2} C\left(\chi_{y x}^{N R}+i \chi_{y x}^{N I}\right) .
\end{gathered}
$$

In these expressions,

$$
C \equiv \frac{1}{36 \omega^{4}} \frac{n_{d 0} a^{3}}{d_{r}^{2}+d_{i}^{2}}
$$

and

where

$$
\begin{aligned}
& \chi_{i j}^{N R}=\hat{A} F_{i j}^{R}-\hat{B} F_{i j}^{I} \\
& \chi_{i j}^{N I}=\hat{B} F_{i j}^{R}+\hat{A} F_{i j}^{I},
\end{aligned}
$$

$$
\begin{aligned}
& \hat{A} \equiv d_{i}+\left(d_{r}-d_{i}\right) \delta_{i j}, \\
& \hat{B} \equiv d_{r}-\left(d_{r}+d_{i}\right) \delta_{i j},
\end{aligned}
$$


$\delta_{i j}$ is the Kronecker delta, and

$$
d_{r}=1-\frac{v_{1}^{I}}{\omega}, \quad d_{i}=\frac{\nu_{d}^{0}}{\omega}+\frac{\nu_{1}^{R}}{\omega},
$$

with

$$
\begin{gathered}
\nu_{d}^{0}=\frac{a}{c(2 \pi)^{1 / 2}}\left(\omega_{p i}^{2} \mu_{i}^{1 / 2}+\omega_{p e}^{2} \mu_{e}^{1 / 2} e^{-Z_{d} e^{2} / T a}\right), \\
v_{1}^{R}=-\frac{A}{\omega}\left(\frac{\pi}{2}\right)^{1 / 2} n_{d 0} a^{3}\left\{\omega_{p i}^{2} \mu_{i}^{3 / 2}\left[I(0,5)+2 \tilde{C}_{i} I(0,3)+\tilde{C}_{i}^{2} I(0,1)\right]\right. \\
\left.+\omega_{p e}^{2} \mu_{e}^{3 / 2}\left[E(0,5)+2 \tilde{C}_{e} E(0,3)+\tilde{C}_{e}^{2} E(0,1)\right]\right\}, \\
v_{1}^{I}=-\frac{1}{\omega}\left(\frac{\pi}{2}\right)^{1 / 2} n_{d 0} a^{3}\left\{\omega_{p i}^{2} \mu_{i}^{3 / 2}\left[I(0,4)+\tilde{C}_{i} I(0,2)\right]\right. \\
\left.+\omega_{p e}^{2} \mu_{e}^{3 / 2}\left[E(0,4)+\tilde{C}_{e} E(0,2)\right]\right\} .
\end{gathered}
$$

The full expressions for the $F_{i j}^{R}$ and $F_{i j}^{I}$ are given in Appendix D.

\section{Numerical results}

In this section, we present a numerical analysis of the effects of the dust particles with variable charge on the propagation properties of the magnetosonic wave. The two main effects to be investigated are those due to the modelling of the charge variation of the dust particles and those due to finiteLarmor-radius effects of electrons and ions. The plasma parameters will be chosen in the range of typical values for laboratory and astrophysical plasmas.

We recall that in paper I, we also analysed the propagation properties of the magnetosonic wave, but restricted to the cold-plasma limit, and using a real wavector $\mathbf{k}$ and a complex frequency $\omega$. In the application developed in paper I, very early on we made the assumption that $\omega \ll \Omega_{i}$, and also made several expansions of denominators of integrands that occur in the components of the dielectric tensor and are currently used in the literature (Prasad 1998). In the present paper, these expansions are not made, and we are interested also in making a comparison between the two ways of calculating the dielectric tensor. The expressions obtained in the present paper for the dispersion relation of the magnetosonic wave are valid for $\omega \gg \Omega_{d}$, because the motion of the dust particles is not considered in the model here proposed.

We note that in Figs 1-6, the frequencies are in the range $\Omega_{d} \ll \omega<\Omega_{i}$, because we are mainly interested in the magnetosonic wave without the effects of finite ion-cyclotron frequency being taken into account; in Figs 7 and 8 we use the range $\Omega_{d} \ll \omega \leqslant 2 \Omega_{i}$, because we are interested in the behaviour of the refractive index for frequencies near the ion-cyclotron frequency.

Initially, we show the results obtained using the cold-plasma approximation and the technique of expansion of the denominators used in paper I, assuming real $\omega$ and complex $k$.

In Figs 1 and 2, we show the real part of the refractive index, $N_{r}=c k_{r} / \omega$, and the complex part, $N_{i}=c k_{i} / \omega$, respectively, as functions of the wave frequency 


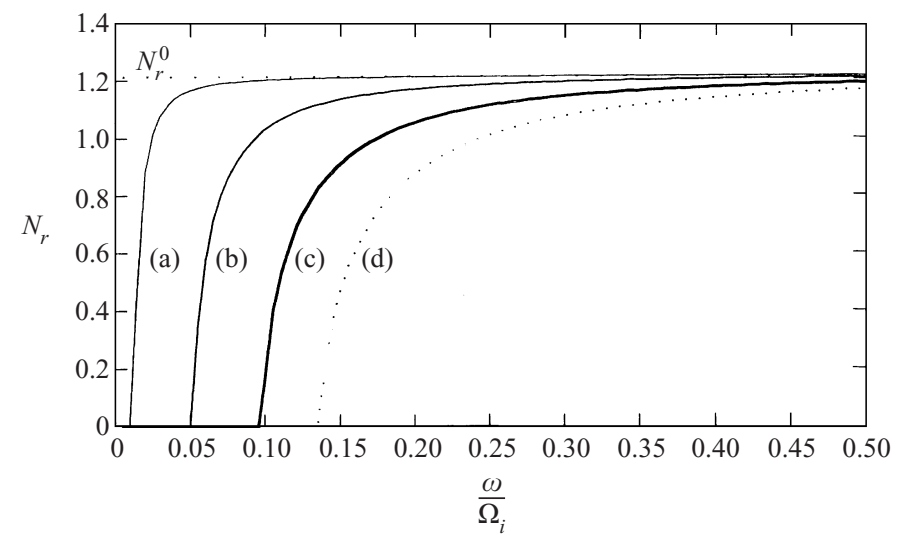

Figure 1. $N_{r}$ as a function of $\omega / \Omega_{i}$, calculated as in paper I, for several values of $T=T_{e}=$ $T_{i}$. Curves (a), (b), (c) and (d) correspond to $T=0.25,1,2$ and $3 \mathrm{eV}$ respectively. The fixed parameters are $n_{i 0}=10^{8} \mathrm{~cm}^{-3}, a=10^{-4} \mathrm{~cm}, n_{d 0}=10^{4} \mathrm{~cm}^{-3}$ and $B_{0}=0.2 \mathrm{~T}$. The curve $N_{r}^{0}$ correspond to the refractive index in the absence of dust particles.

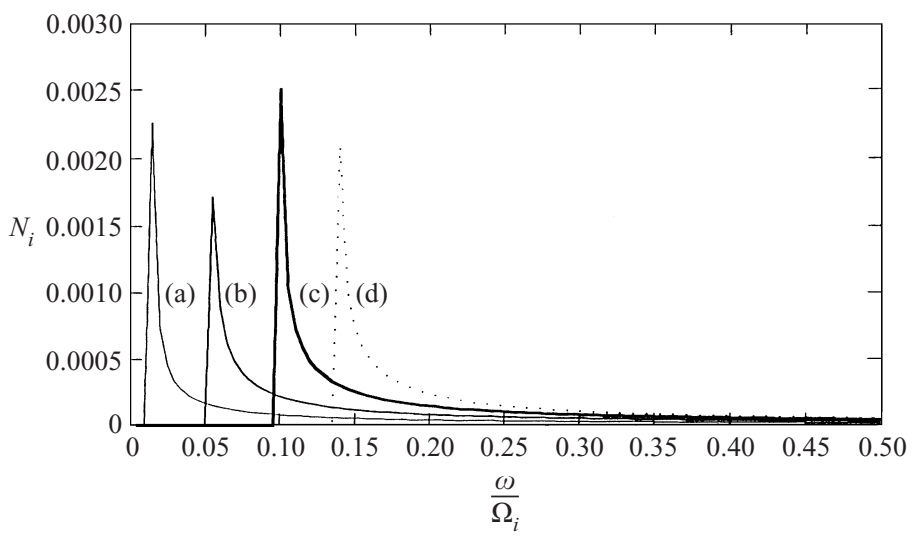

Figure 2. $N_{i}$ as a function of $\omega / \Omega_{i}$, calculated as in paper I, for several values of $T=T_{e}=$ $T_{i}$. Curves (a), (b), (c) and (d) correspond to $T=0.25,1,2$ and $3 \mathrm{eV}$ respectively, and the other parameters are the same as in Fig. 1.

normalized to the ion-cyclotron frequency $\omega / \Omega_{i}$, for several values of the temperature $T=T_{e}=T_{i}$. Curves (a), (b), (c) and (d) correspond to $T=0.25,1$, 2 and $3 \mathrm{eV}$ respectively. The fixed parameters are $n_{i 0}=10^{8} \mathrm{~cm}^{-3}, a=10^{-4} \mathrm{~cm}$, $n_{d 0}=10^{4} \mathrm{~cm}^{-3}$ and $B_{0}=0.2 \mathrm{~T}$. These figures are obtained from the following expressions derived from paper I:

$$
\begin{gathered}
N_{r}^{2}=\mathscr{A}[1-F(\omega)], \\
2 N_{r} N_{i}=\frac{\mathscr{B}}{\omega}[1+F(\omega)],
\end{gathered}
$$

where

$$
F(\omega)=\frac{\mathscr{C}^{2}}{\mathscr{A}^{2} \omega^{2}+\mathscr{B}^{2}},
$$


and

$$
\begin{gathered}
\mathscr{A} \equiv 1+\sum_{\beta} \frac{\omega_{p \beta}^{2}}{\Omega_{\beta}^{2}} \approx 1+\frac{c^{2}}{V_{A i}^{2}}, \\
\mathscr{B} \equiv \frac{4 a^{2} n_{d 0}}{3} \sqrt{2 \pi} \sum_{\beta} \frac{\omega_{p \beta}^{2}}{\Omega_{\beta}^{2}} u_{T \beta}\left(2+\frac{Z_{d} e^{2}}{T a}\right)\left[\delta_{\beta i}+\exp \left(-\frac{Z_{d} e^{2}}{T a}\right) \delta_{\beta e}\right], \\
\mathscr{C} \equiv \sum_{\beta} \frac{\omega_{p \beta}^{2}}{\Omega_{\beta}}=\frac{4 \pi}{B_{0}} Z_{d} e n_{d 0} .
\end{gathered}
$$

The cutoff of the real part of the refractive index, which appears in Fig. 1, occurs when $F(\omega)=1$, which gives for the cutoff frequency $\omega_{c}$ the value

$$
\left(\frac{\omega_{c}}{\Omega_{i}}\right)^{2}=\frac{\mathscr{C}^{2}-\mathscr{B}^{2}}{\mathscr{A}^{2} \Omega_{i}^{2}} .
$$

Because in this expression only $\mathscr{B}$ depends on the temperature and this dependence is approximately proportional to $1 / T$, when $T$ increases, $\mathscr{B}$ diminishes and $\omega_{c}$ increases, in agreement with Fig. 1.

When the dust particles are absent $\left(n_{d 0}=0\right)$ or when their charges are fixed $(\mathscr{B}=0)$, we have

$$
N_{r}^{0}=\mathscr{A}^{1 / 2} \approx\left(1+\frac{4 \pi c^{2} n_{i 0} m_{i}}{B_{0}}\right)^{1 / 2},
$$

where $N_{r}^{0}$ indicates the real part of the refractive index in the absence of dust. In Fig. 1, we see that at high frequencies, $N_{r}$ tends to $N_{r}^{0}$. In this case, $N_{i}^{0}=0$, as expected.

The inclusion of charged dust particles with variable charge in the plasma results in $N_{i} \neq 0$ and $N_{i}>0$, which implies that the wave is spatially absorbed when travelling in the plasma.

We have also estimated the dependence on temperature of quantities that play an important role in the physical description of the process we are studying. The frequencies $v_{e d}$ and $v_{i d}$, characterizing the rate of capture of electrons and ions respectively by dust particles in the equilibrium state, and also $Z_{d}$, increase with temperature, but $n_{e 0}$ and the charging frequency $\nu_{d}^{0}$ decrease with temperature. For the parameters used in Figs 1 and 2, the values of $v_{e d}^{0} / \Omega_{i}$ and $\nu_{d}^{0} / \Omega_{i}$ are always localized to the left of the corresponding cutoff frequency. In other words, for $\omega<\omega_{c}$, we have the possibility that $\omega$ can be less than $\nu_{d}^{0}$ and $\nu_{\beta d}^{0}$, which implies that in this range of frequencies, the condition $\omega \gg \max \left(k v_{T e}, v_{d}^{0}, v_{\beta d}^{0}\right)$ is not fulfilled. Then the expansion of denominators that has been made is very bad in this region of the frequency spectrum, and probably leads to the cutoff obtained.

In Figs 3 and 4, we compare numerical results obtained using the expressions for the refractive index of the magnetosonic wave from paper I with those obtained in this paper, in the cold-plasma approximation.

In Fig. 3, the real part of the refractive index in the cold-plasma model is plotted as a function of $\omega / \Omega_{i}$ using the two procedures for the calculation. The straight line shows $N_{r}=N_{r}^{0}$ in the cold-plasma model for a plasma without dust. Curve (a) shows $N_{r}$ calculated using the procedure developed in this paper; curve (b) shows $N_{r}$ as calculated in paper I. The parameters used are $a=$ 


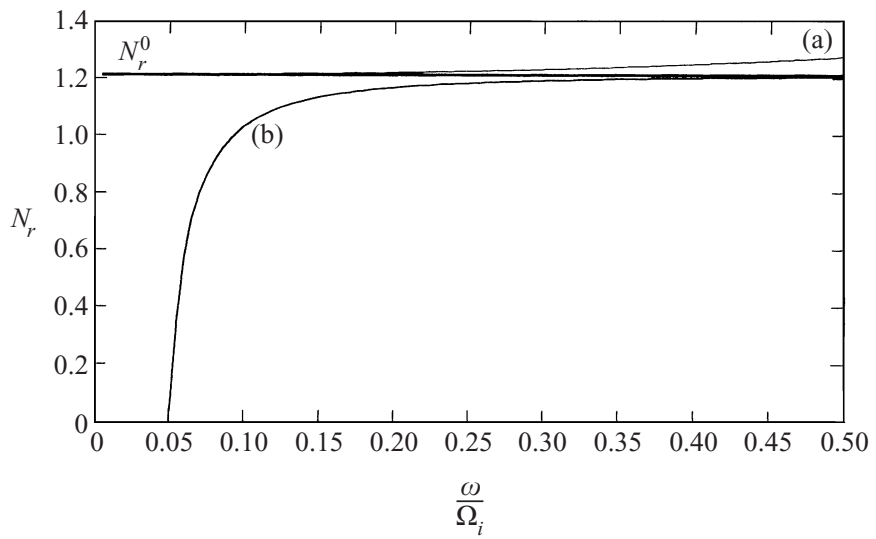

Figure 3. $N_{r}$ as a function of $\omega / \Omega_{i}$, using the cold-plasma model, the parameters being $a=$ $10^{-4} \mathrm{~cm}, n_{i 0}=10^{8} \mathrm{~cm}^{-3}, n_{d 0}=10^{4} \mathrm{~cm}^{-3}, B_{0}=0.2 \mathrm{~T}$ and $T=1 \mathrm{eV}$. Curve (a) was calculated using the procedure developed in this paper, while curve (b) corresponds to the calculation using the procedure of paper I. The curve $N_{r}^{0}$ corresponds to the refractive index in the absence of dust particles.

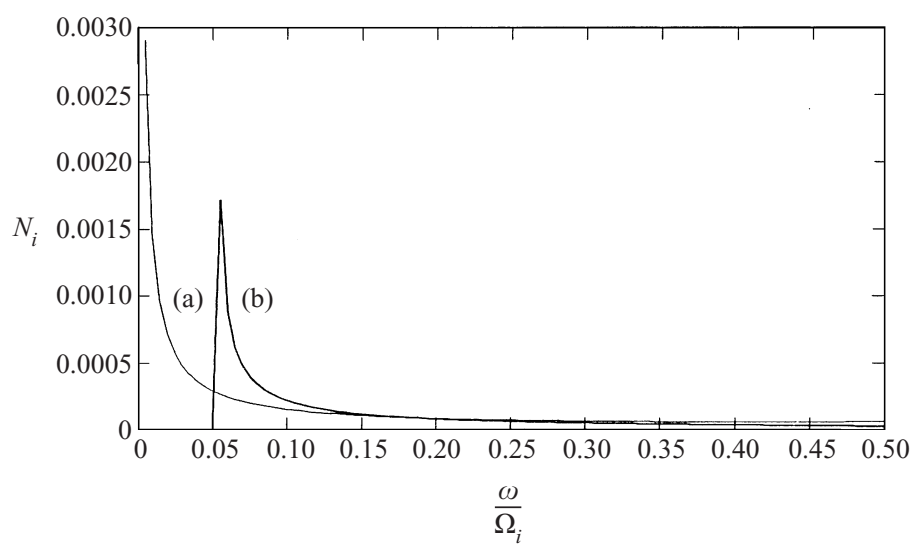

Figure 4. $N_{i}$ as a function of $\omega / \Omega_{i}$, using the cold-plasma model, for the same parameters and situations presented in Fig. 3 .

$10^{-4} \mathrm{~cm}, n_{i 0}=10^{8} \mathrm{~cm}^{-3}, n_{d 0}=10^{4} \mathrm{~cm}^{-3}, B_{0}=0.2 \mathrm{~T}$ and $T=1 \mathrm{eV}$. For this set of parameters, we see that the cutoff, which originates from the formulation developed in paper I, does not occur in the region $\Omega_{d} / \Omega_{i} \ll \omega / \Omega_{i} \ll 1$ when we use the procedure developed in this paper. We can say that the approach of paper I is satisfactory for situations where the temperature is not very high, and that the region of agreement between the two methods of calculating $N_{r}$ increases as the temperature is lowered.

$N_{r}$ obtained from the calculations of this paper, represented by curve (a), departs from the value of $N_{r}^{0}$ when $\omega / \Omega_{i}$ increases. The results from paper I are unable to represent this feature, because we have assumed from the start that $\omega / \Omega_{i} \ll 1$, in order to make the expansions of the denominators.

In Fig. 4, we present, for the same parameters and situations used in Fig. 3, $N_{i}$ as a function of $\omega / \Omega_{i}$. The cutoff exhibited by $N_{r}$ generates a forbidden region of values of $N_{i}$ in curve (b) obtained from paper I, which does not exist in curve 


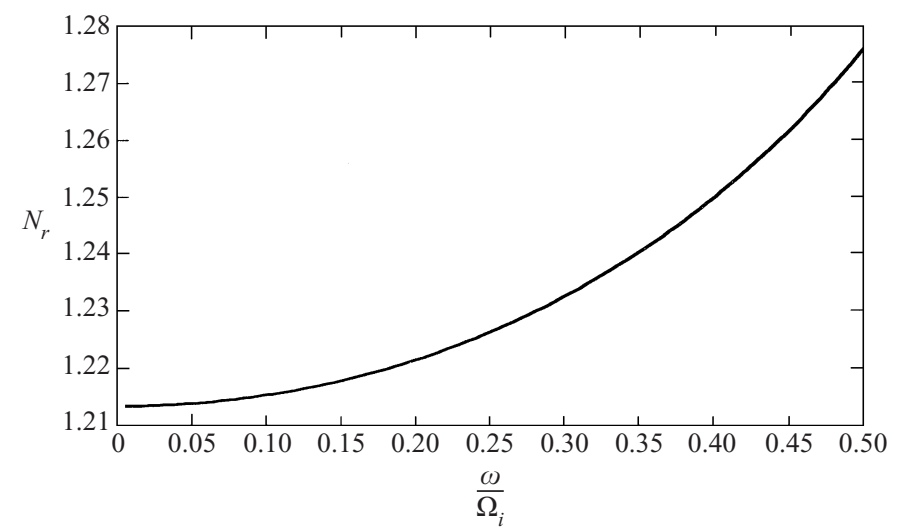

Figure 5. $N_{r}$ as a function of $\omega / \Omega_{i}$, including Larmor-radius effects. The parameters used are $n_{d 0}=10^{4} \mathrm{~cm}^{-3}, n_{i 0}=10^{8} \mathrm{~cm}^{-3}, a=10^{-4} \mathrm{~cm}$ and $B_{0}=0.2 \mathrm{~T}$. The same curve is obtained for $T=0.06,0.25$ and $1 \mathrm{eV}$.

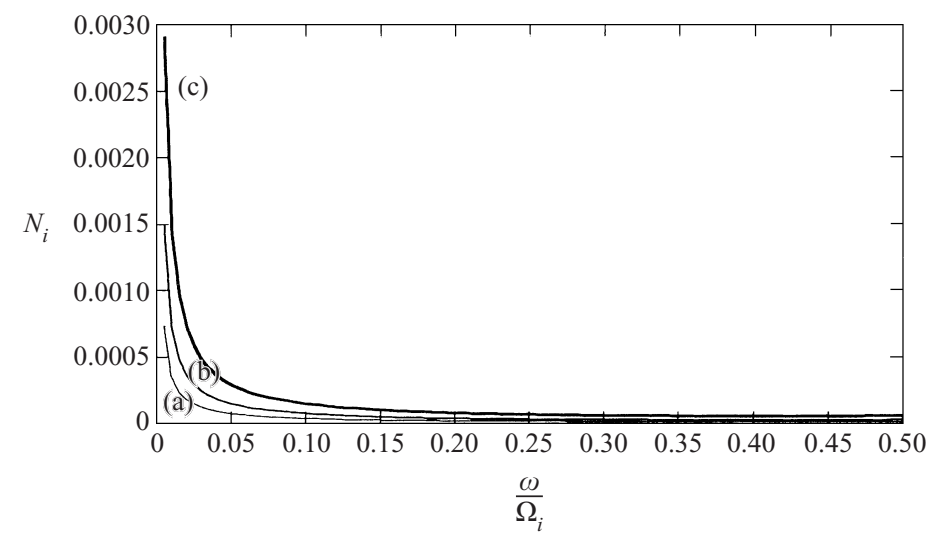

Figure 6. $N_{i}$ as function of $\omega / \Omega_{i}$, including Larmor-radius effects, using the same parameters as in Fig. 5. The curves labelled by (a), (b) and (c) correspond to $T=0.06,0.25$ and $1 \mathrm{eV}$ respectively.

(a). We notice that this result comes from a formulation in which the expansion of the denominators has not been introduced, and in which, therefore, the effects of charge variation are dealt with in a more complete way. Figure 4 shows that the charge variation of the dust particles is more important for lower values of $\omega / \Omega_{i}$.

In Figs 5 and 6 , we show $N_{r}$ and $N_{i}$ as functions of $\omega / \Omega_{i}$, calculated using the procedure developed in this paper and including Larmor-radius effects of electrons and ions, for $\Omega_{d} \ll \omega<\Omega_{i}$. The parameters used are $n_{d 0}=10^{4} \mathrm{~cm}^{-3}$, $n_{i 0}=10^{8} \mathrm{~cm}^{-3}, a=10^{-4} \mathrm{~cm}$ and $B_{0}=0.2 \mathrm{~T}$, and the temperatures are (a) $T=0.06 \mathrm{eV}$, (b) $T=0.25 \mathrm{eV}$ and (c) $T=1 \mathrm{eV}$.

We see in Fig. 5 that for this set of temperature values, we obtain approximately the same curve for $N_{r}$, which departs only slightly, for low values of $\omega / \Omega_{i}$, from the straight line that characterizes the plasma without dust. We can say that $N_{r}$ is not very sensitive to the charge variation of the dust particles, which is influenced by the temperature, which in turn modifies the frequencies 


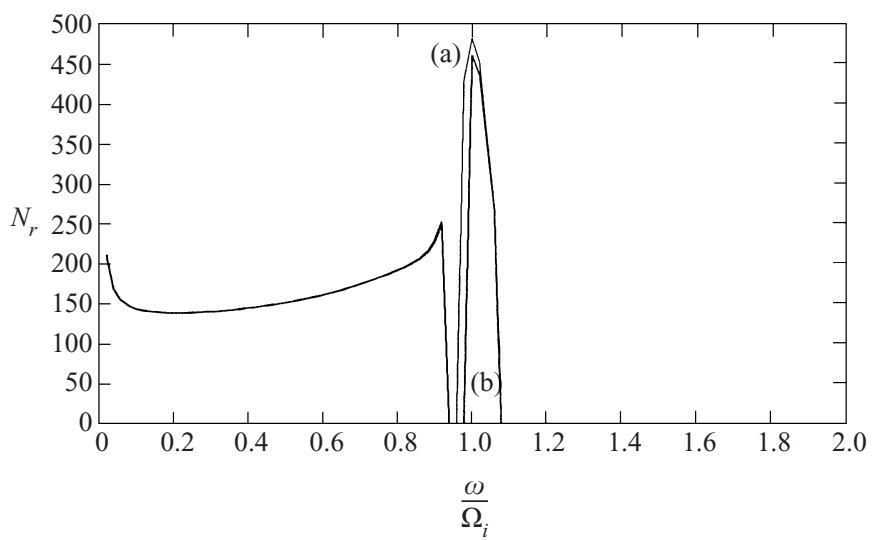

Figure 7. $N_{r}$ as a function of $\omega / \Omega_{i}$, using the expressions developed in this paper: curve (a) includes Larmor-radius effects; curve $(b)$ is for the cold-plasma model. The parameters used are $n_{i 0}=10^{8} \mathrm{~cm}^{-3}, a=10^{-4} \mathrm{~cm}, n_{d 0}=10^{4} \mathrm{~cm}^{-3}, T=100 \mathrm{eV}$ and $B_{0}=10^{-3} \mathrm{~T}$.

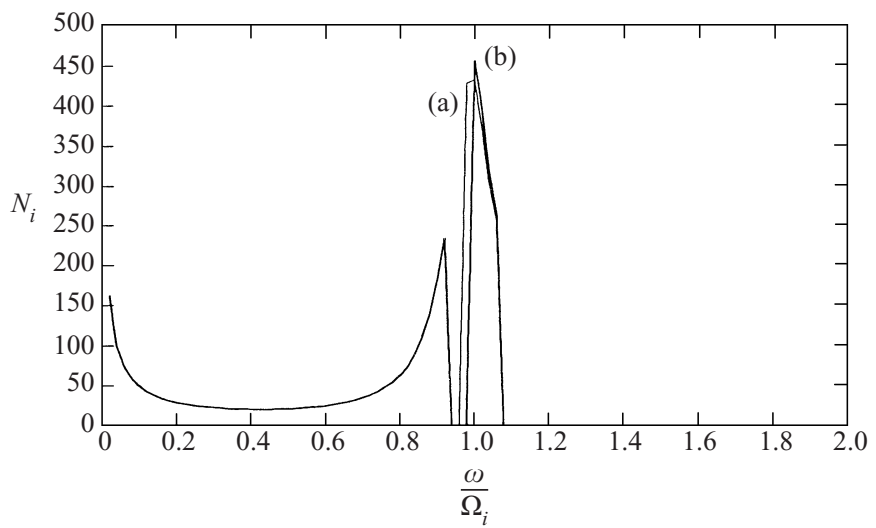

Figure 8. $N_{i}$ as a function of $\omega / \Omega_{i}$ for the same situations and parameters as in Fig. 7 .

related to the charging process and also the charging time. We expect that in the frequency region where $\omega \ll \Omega_{d}$, $N_{r}$ will be altered more significantly when the temperature is changed, because it is in this region that the characteristic charging frequencies are localized. Also in this frequency region, new wave propagation modes (Rao 1995) will arise owing to the inclusion of the motion of dust particles.

In Fig. 6, we see that for fixed values of $\omega / \Omega_{i}, N_{i}$ increases with increasing temperature. For $\omega / \Omega_{i}>0.2$, the values of $N_{i}$ are approximately equal and very small. Again we observe that the influence of the charge variation of the dust particles is enhanced at low frequencies. We remark that when $\omega$ increases relative to the charging frequency $v_{d}^{0}$, the importance of the charge variation of the dust particles decreases, and accordingly $N_{i}$ must decrease, because it is directly connected with charge variation.

From the numerical calculation, we observe that the Larmor-radius effects included are negligible in the range of frequencies $\Omega_{d} \ll \omega<\Omega_{i}$.

In Figs 7 and 8, we extend the region of variation of the frequency to include frequencies above $\Omega_{i}$, and compare, using the procedure developed in this paper 
to calculate the dielectric tensor, the situation of a cold plasma with a situation where Larmor-radius effects are retained.

In Figs 7 and 8, we show $N_{r}$ and $N_{i}$ respectively as functions of $\omega / \Omega_{i}$, with curve (a) corresponding to the situation with Larmor-radius effects included and curve (b) to the cold-plasma limit. The parameters used are $n_{\mathrm{i} 0}=10^{8} \mathrm{~cm}^{-3}$, $a=10^{-4} \mathrm{~cm}, n_{d 0}=10^{4} \mathrm{~cm}^{-3}, T=100 \mathrm{eV}$ and $B_{0}=10^{-3} \mathrm{~T}$.

We observe that in the range of frequencies $\omega<\Omega_{i}$, Larmor-radius effects are unimportant, but in the range $\omega \geqslant \Omega_{i}$, they start to contribute significantly, because for each component of the dielectric tensor, the quantities $\chi_{i j}^{0 R} / \chi_{i j}^{1 R}$ and $\chi_{i j}^{0 I} / \chi_{i j}^{1 I}$, which characterize the relative importance of powers of the Larmor radius, are proportional to $Y_{\beta} \equiv \Omega_{\beta} / \omega$.

From the numerical calculation, we observe that the dominant contributions of the Larmor radius arise from the 'homogeneous' part of the dielectric tensor $\left(\epsilon_{i j}^{h}\right)$. The contributions of order $N^{2}$ arising from $\epsilon_{i j}^{N}$ are, for the situations tested, negligible - essentially because the coefficient $C$, which is a multiplicative factor for all components, is very small.

\section{Conclusions}

Although in the present paper we have concentrated on the study of a magnetosonic wave, which implies perpendicular propagation, the results of Sect. 2 apply to all directions of propagation. We expect that the contributions due to the charge variation of the dust particles of order $N^{2}$, included in $\epsilon_{i j}^{N}$, can be relevant for parallel propagation of the wave.

Also, if we include the effects of inhomogeneities in the dielectric tensor, we can study the influence of the dust particles with variable charge on Alfvénwave resonant absorption and in its conversion to a kinetic Alfvén wave. The modification of the Alfvén resonance absorption mechanism due to the presence of charged dust grains was considered by Cramer and Vladimirov (1998). Damping due to the charging of the grains was included in such a way that this corresponds only to the effects included in the part $\tilde{\epsilon}_{i j}^{h}$ of the dielectric tensor, presented here. For instance, Hasegawa (1976) pointed out that in regions near the resonant point, Larmor-radius effects become important. This suggests that the effects of the charging of the grains included in the part $\tilde{\epsilon}_{i j}^{N}$ of our tensor would be essential in studying Alfvén resonance absorption. We intend to include the effects of inhomogeneities in our tensor and to apply it to this topic in the future.

\section{Appendix A}

In Appendix A of paper I, we presented the quantities $R_{i j}^{n \beta}$ in their general form, as functions of $\psi$, the angle between the vector $\mathbf{k}_{\perp}$ and the $x$ axis. For the case of $\psi=0$, considered in the present paper, the quantities $R_{i j}^{n \beta}$ assume the following forms :

$$
\begin{gathered}
R_{x x}^{n \beta}=\frac{n^{2}}{b_{\beta}^{2}} J_{n}^{2}\left(b_{\beta}\right), \\
R_{x y}^{n \beta}=-R_{y x}^{n \beta}=i \frac{n}{b_{\beta}} J_{n}\left(b_{\beta}\right) J_{n}^{\prime}\left(b_{\beta}\right),
\end{gathered}
$$


where $b_{\beta}=k_{\perp} c u_{\perp} / \Omega_{\beta}$.

$$
\begin{gathered}
R_{x z}^{n \beta}=R_{z x}^{n \beta}=\frac{n}{b_{\beta}} J_{n}^{2}\left(b_{\beta}\right), \\
R_{y y}^{n \beta}=J_{n}^{\prime 2}\left(b_{\beta}\right), \\
R_{y z}^{n \beta}=-R_{z y}^{n \beta}=-i J_{n}\left(b_{\beta}\right) J_{n}^{\prime}\left(b_{\beta}\right), \\
R_{z z}^{n \beta}=J_{n}^{2}\left(b_{\beta}\right),
\end{gathered}
$$

\section{Appendix B}

The components of the dielectric tensor $\epsilon_{i j}=\tilde{\epsilon}_{i j}^{h}+\epsilon_{i j}^{N}$, in terms of the integrals $\tilde{I}_{\beta}(n, m, h, s, G)$, are given in the following. The $\tilde{\epsilon}_{i j}^{h}$ components are written as

$$
\begin{aligned}
& \tilde{\epsilon}_{x x}^{h}=1+\sum_{\beta} X_{\beta} \sum_{n=1}^{\infty} \sum_{m=0}^{\infty}\left(\frac{N_{\perp}}{Y_{\beta}}\right)^{2(n+m-1)} n^{2} a(n, m) \\
& \times \sum_{s= \pm 1} \tilde{I}_{\beta}\left(n, m, 0, s ; \mathscr{L} F_{\beta 0}\right), \\
& \tilde{\epsilon}_{x y}^{h}=-i \sum_{\beta} X_{\beta} \sum_{n=1}^{\infty} \sum_{m=0}^{\infty}\left(\frac{N_{\perp}}{Y_{\beta}}\right)^{2(n+m-1)} n(n+m) a(n, m) \\
& \times \sum_{s= \pm 1} s \tilde{I}_{\beta}\left(n, m, 0, s ; \mathscr{L} F_{\beta 0}\right), \\
& \tilde{\epsilon}_{x z}^{h}=-\sum_{\beta} X_{\beta} \frac{N_{\perp}}{Y_{\beta}} \sum_{n=1}^{\infty} \sum_{m=0}^{\infty}\left(\frac{N_{\perp}}{Y_{\beta}}\right)^{2(n+m-1)} n a(n, m) \\
& \times \sum_{s= \pm 1} s\left[\tilde{I}_{\beta}\left(n, m, 1, s ; \mathscr{L} F_{\beta 0}\right)+\tilde{I}_{\beta}\left(n, m, 0, s ; \frac{i v_{\beta d}^{0}}{\omega} L F_{\beta 0}\right)\right], \\
& \tilde{\epsilon}_{y y}^{h}=1+\sum_{\beta} X_{\beta} \sum_{m=2}^{\infty}\left(\frac{N_{\perp}}{Y_{\beta}}\right)^{2(m-1)} d(0, m) \tilde{I}_{\beta}\left(0, m, 0,0 ; \mathscr{L} F_{\beta 0}\right) \\
& +\sum_{\beta} X_{\beta} \sum_{n=1}^{\infty} \sum_{m=0}^{\infty}\left(\frac{N_{\perp}}{Y_{\beta}}\right)^{2(n+m-1)} d(n, m) \sum_{s= \pm 1} \tilde{I}_{\beta}\left(n, m, 0, s ; \mathscr{L} F_{\beta 0}\right), \\
& \tilde{\epsilon}_{y z}^{h}=-i \sum_{\beta} X_{\beta} \frac{N_{\perp}}{Y_{\beta}} \sum_{m=1}^{\infty}\left(\frac{N_{\perp}}{Y_{\beta}}\right)^{2(m-1)} m a(0, m) \\
& \times\left[\tilde{I}_{\beta}\left(0, m, 1,0 ; \mathscr{L} F_{\beta 0}\right)+\tilde{I}_{\beta}\left(0, m, 0, s ; \frac{i v_{\beta d}^{0}}{\omega} L F_{\beta 0}\right)\right] \\
& -i \sum_{\beta} X_{\beta} \frac{N_{\perp}}{Y_{\beta}} \sum_{n=1}^{\infty} \sum_{m=0}^{\infty}\left(\frac{N_{\perp}}{Y_{\beta}}\right)^{2(n+m-1)}(n+m) a(n, m) \\
& \times \sum_{s= \pm 1}\left[\tilde{I}_{\beta}\left(n, m, 1, s ; \mathscr{L} F_{\beta 0}\right)+\tilde{I}_{\beta}\left(n, m, 0, s ; \frac{i v_{\beta d}^{0}}{\omega} L F_{\beta 0}\right)\right],
\end{aligned}
$$




$$
\begin{aligned}
\tilde{\epsilon}_{z z}^{h}= & +\sum_{\beta} X_{\beta} \tilde{I}_{\beta}\left(0,0,1,0 ; u_{\perp} \frac{\partial F_{\beta 0}}{\partial u_{\|}}\right) \\
& +\sum_{\beta} X_{\beta} \sum_{m=1}^{\infty}\left(\frac{N_{\perp}}{Y_{\beta}}\right)^{2 m} a(0, m) \\
& \times\left[\tilde{I}_{\beta}\left(0, m, 2,0 ; \mathscr{L} F_{\beta 0}\right)+\tilde{I}_{\beta}\left(0, m, 1,0 ; \frac{i v_{\beta d}^{0}}{\omega} L F_{\beta 0}\right)\right] \\
& +\sum_{\beta} X_{\beta} \sum_{n=1}^{\infty} \sum_{m=0}^{\infty}\left(\frac{N_{\perp}}{Y_{\beta}}\right)^{2(n+m)} a(n, m) \\
& \times \sum_{s= \pm 1}\left[\tilde{I}_{\beta}\left(n, m, 2, s ; \mathscr{L} F_{\beta 0}\right)+\tilde{I}_{\beta}\left(n, m, 1, s ; \frac{i v_{\beta d}^{0}}{\omega} L F_{\beta 0}\right)\right],
\end{aligned}
$$

and $\tilde{\epsilon}_{j i}^{h}=\left(\tilde{\epsilon}_{i j}^{h}\right)^{*}$.

Similarly, the $\epsilon_{i j}^{N}$ components are written as

$$
\epsilon_{i j}^{N}=-\frac{i}{\omega} \frac{c^{2}}{\omega^{2}} \frac{1}{1+i\left(\frac{\nu_{d}^{0}}{\omega}+\frac{\nu_{1}}{\omega}\right)} U_{i} S_{j},
$$

where

$$
\begin{gathered}
U_{x}=-\sum_{\beta} q_{\beta} n_{\beta 0} \sum_{n=1}^{\infty} \sum_{m=0}^{\infty} n a(n, m)\left(\frac{N_{\perp}}{Y_{\beta}}\right)^{2(n+m)-1} \\
\times \sum_{s= \pm 1} s \tilde{I}_{\beta}\left(n, m, 0, s ; u \sigma_{\beta}^{\prime} u_{\perp} F_{\beta 0}\right), \\
U_{y}=-i \sum_{\beta} q_{\beta} n_{\beta 0}\left[\sum_{m=1}^{\infty} m a(0, m)\left(\frac{N_{\perp}}{Y_{\beta}}\right)^{2 m-1} \tilde{I}_{\beta}\left(0, m, 0,0 ; u \sigma_{\beta}^{\prime} u_{\perp} F_{\beta 0}\right)\right. \\
\left.+\sum_{n=1}^{\infty} \sum_{m=0}^{\infty}(n+m) a(n, m)\left(\frac{N_{\perp}}{Y_{\beta}}\right)^{2(n+m)-1} \sum_{s= \pm 1} \tilde{I}_{\beta}\left(n, m, 0, s ; u \sigma_{\beta}^{\prime} u_{\perp} F_{\beta 0}\right)\right], \\
U_{z}=\sum_{\beta} q_{\beta} n_{\beta 0}\left[\sum_{m=0}^{\infty} a(0, m)\left(\frac{N_{\perp}}{Y_{\beta}}\right)^{2 m} \tilde{I}_{\beta}\left(0, m, 1,0 ; u \sigma_{\beta}^{\prime} u_{\perp} F_{\beta 0}\right)\right. \\
\left.+\sum_{n=1}^{\infty} \sum_{m=0}^{\infty} a(n, m)\left(\frac{N_{\perp}}{Y_{\beta}}\right)^{2(n+m)} \sum_{s= \pm 1} \tilde{I}_{\beta}\left(n, m, 1, s ; u \sigma_{\beta}^{\prime} u_{\perp} F_{\beta 0}\right)\right] \\
S_{x}=-\sum_{\beta} \frac{\omega_{p \beta}^{2}}{c} \sum_{n=1}^{\infty} \sum_{m=0}^{\infty} n a(n, m)\left(\frac{N_{\perp}}{Y_{\beta}}\right)^{2(n+m)-1} \\
\quad \times \sum_{s= \pm 1} s \tilde{I}_{\beta}\left(n, m, 0, s ; i \frac{\nu_{\beta d}^{0}}{\omega} \gamma_{\beta} \mathscr{L} F_{\beta 0}\right),
\end{gathered}
$$




$$
\begin{aligned}
S_{y}= & i \sum_{\beta} \frac{\omega_{p \beta}^{2}}{c}\left[\sum_{m=1}^{\infty} m a(0, m)\left(\frac{N_{\perp}}{Y_{\beta}}\right)^{2 m-1} \tilde{I}_{\beta}\left(0, m, 0,0 ; i \frac{\nu_{\beta d}^{0}}{\omega} \gamma_{\beta} \mathscr{L} F_{\beta 0}\right)\right. \\
& +\sum_{n=1}^{\infty} \sum_{m=0}^{\infty}(n+m) a(n, m)\left(\frac{N_{\perp}}{Y_{\beta}}\right)^{2(n+m)-1} \\
& \left.\times \sum_{s= \pm 1} \tilde{I}_{\beta}\left(n, m, 0, s ; i \frac{\nu_{\beta d}^{0}}{\omega} \gamma_{\beta} \mathscr{L} F_{\beta 0}\right)\right], \\
S_{z}= & \sum_{\beta} \frac{\omega_{p \beta}^{2}}{c}\left[\tilde{I}_{\beta}\left(0,0,0,0 ; i \frac{\nu_{\beta d}^{0}}{\omega} u_{\perp} \frac{\partial F_{\beta 0}}{\partial u_{\|}}\right)-2 \tilde{I}_{\beta}\left(0,0,0,0 ;\left(i \frac{\nu_{\beta d}^{0}}{\omega}\right)^{2} L F_{\beta 0}\right)\right] \\
+ & \sum_{\beta} \frac{\omega_{p \beta}^{2}}{c} \sum_{m=1}^{\infty}\left(\frac{N_{\perp}}{Y_{\beta}}\right)^{2 m} a(0, m) \\
\times & {\left[\tilde{I}_{\beta}\left(0, m, 1,0 ; i \frac{\nu_{\beta d}^{0}}{\omega} \mathscr{L} F_{\beta 0}\right)-\tilde{I}_{\beta}\left(0, m, 0,0 ;\left(i \frac{\nu_{\beta d}^{0}}{\omega}\right)^{2} L F_{\beta 0}\right)\right] } \\
+ & \sum_{\beta} \frac{\omega_{p \beta}^{2}}{c} \sum_{n=1}^{\infty} \sum_{m=0}^{\infty}\left(\frac{N_{\perp}}{Y_{\beta}}\right)^{2(n+m)} a(n, m) \\
\times & \sum_{s= \pm 1}\left[\tilde{I}_{\beta}\left(n, m, 1, s ; i \frac{\nu_{\beta d}^{0}}{\omega} \mathscr{L} F_{\beta 0}\right)-\tilde{I}_{\beta}\left(n, m, 0, s ;\left(i \frac{\nu_{\beta d}^{0}}{\omega}\right)^{2} L F_{\beta 0}\right)\right] .
\end{aligned}
$$

The parameter $v_{1}$ is given by

$$
\begin{aligned}
\nu_{1}= & c \sum_{\beta} q_{\beta} n_{\beta 0}\left[\sum_{m=0}^{\infty} a(0, m)\left(\frac{N_{\perp}}{Y_{\beta}}\right)^{2 m} \tilde{I}_{\beta}\left(0, m, 0,0 ; i \frac{\nu_{\beta d}^{0}}{\omega} \gamma_{\beta} u_{\perp} u \sigma_{\beta}^{\prime} F_{\beta 0}\right)\right. \\
& \left.+\sum_{n=1}^{\infty} \sum_{m=0}^{\infty} a(n, m)\left(\frac{N_{\perp}}{Y_{\beta}}\right)^{2(n+m)} \sum_{s= \pm 1} \tilde{I}_{\beta}\left(n, m, 0, s ; i \frac{\nu_{\beta d}^{0}}{\omega} \gamma_{\beta} u_{\perp} u \sigma_{\beta}^{\prime} F_{\beta 0}\right)\right],
\end{aligned}
$$

and $\nu_{d}^{0}$ is given by $(2.9)$.

\section{Appendix C}

The expressions for $\chi_{i j}^{0 R}, \chi_{i j}^{0 I}, \chi_{i j}^{1 R}$ and $\chi_{i j}^{1 I}$ are as follows:

$$
\begin{aligned}
\chi_{x x}^{0 R}= & -\frac{1}{3(2 \pi)^{1 / 2}}\left\{X_{i} \mu_{i}^{5 / 2}\left[\left(1+Y_{i}\right) I(1,6)+\left(1-Y_{i}\right) I(-1,6)\right]\right. \\
& \left.+X_{e} \mu_{e}^{5 / 2}\left[\left(1+Y_{e}\right) E(1,6)+\left(1-Y_{e}\right) E(-1,6)+\frac{2}{1-Y_{e}^{2}} i_{4}\right]\right\} \\
\chi_{x x}^{1 R}= & -\frac{1}{15(2 \pi)^{1 / 2}}\left\{\frac { X _ { i } \mu _ { i } ^ { 5 / 2 } } { Y _ { i } ^ { 2 } } \left[\left(1+2 Y_{i}\right) I(2,8)+\left(1-2 Y_{i}\right) I(-2,8)\right.\right. \\
& \left.-\left(1+Y_{i}\right) I(1,8)-\left(1-Y_{i}\right) I(-1,8)\right] \\
& +\frac{X_{e} \mu_{e}^{5 / 2}}{Y_{e}^{2}}\left[\left(1+2 Y_{e}\right) E(2,8)+\left(1-2 Y_{e}\right) E(-2,8)+\frac{2}{1-4 Y_{e}^{2}} i_{6}-\frac{2}{1-Y_{e}^{2}} i_{6}\right. \\
& \left.\left.-\left(1+Y_{e}\right) E(1,8)-\left(1-Y_{e}\right) E(-1,8)\right]\right\}
\end{aligned}
$$




$$
\begin{aligned}
& \chi_{x x}^{0 I}=\frac{A}{3(2 \pi)^{1 / 2}}\left\{X_{i} \mu_{i}^{5 / 2}\left[I(1,7)+\tilde{C}_{i} I(1,5)+I(-1,7)+\tilde{C}_{i} I(-1,5)\right]\right. \\
& \left.+X_{e} \mu_{e}^{5 / 2}\left[E(1,7)+\tilde{C}_{e} E(1,5)+E(-1,7)+\tilde{C}_{e} E(-1,5)\right]\right\}, \\
& \chi_{x x}^{1 I}=\frac{A}{15(2 \pi)^{1 / 2}}\left\{\frac { X _ { i } \mu _ { i } ^ { 5 / 2 } } { Y _ { i } ^ { 2 } } \left[I(2,9)+\tilde{C}_{i} I(2,7)+I(-2,9)+\tilde{C}_{i} I(-2,7)\right.\right. \\
& \left.-I(1,9)-\tilde{C}_{i} I(1,7)-I(-1,9)-\tilde{C}_{i} I(-1,7)\right] \\
& +\frac{X_{e} \mu_{e}^{5 / 2}}{Y_{e}^{2}}\left[E(2,9)+\tilde{C}_{e} E(2,7)+E(-2,9)+\tilde{C}_{e} E(-2,7)\right. \\
& \left.\left.-E(1,9)-\tilde{C}_{e} E(1,7)-E(-1,9)-\tilde{C}_{e} E(-1,7)\right]\right\}, \\
& \chi_{y y}^{1 R}=-\frac{2}{15(2 \pi)^{1 / 2}}\left\{\frac{X_{i} \mu_{i}^{5 / 2}}{Y_{i}^{2}}\left[2 I(0,8)-\left(1+Y_{i}\right) I(1,8)-\left(1-Y_{i}\right) I(-1,8)\right]\right. \\
& +\frac{X_{e} \mu_{e}^{5 / 2}}{Y_{e}^{2}}\left[2 E(0,8)-\left(1+Y_{e}\right) E(1,8)-\left(1-Y_{e}\right) E(-1,8)\right. \\
& \left.\left.+2 i_{6}-\frac{2}{1-Y_{e}^{2}} i_{6}\right]\right\} \\
& \chi_{y y}^{1 I}=\frac{2 A}{15(2 \pi)^{1 / 2}}\left\{\frac { X _ { i } \mu _ { i } ^ { 5 / 2 } } { Y _ { i } ^ { 2 } } \left[2\left(I(0,9)+\tilde{C}_{i} I(0,7)\right)-I(1,9)-\tilde{C}_{i} I(1,7)\right.\right. \\
& \left.-I(-1,9)-\tilde{C}_{i} I(-1,7)\right] \\
& +\frac{X_{e} \mu_{e}^{5 / 2}}{Y_{e}^{2}}\left[2\left(E(0,9)+\tilde{C}_{e} E(0,7)\right)-E(1,9)-\tilde{C}_{e} E(1,7)\right. \\
& \left.\left.-E(-1,9)-\tilde{C}_{e} E(-1,7)\right]\right\} \text {, } \\
& \chi_{x y}^{0 R}=\frac{A}{3(2 \pi)^{1 / 2}}\left\{X_{i} \mu_{i}^{5 / 2}\left[I(1,7)+\tilde{C}_{i} I(1,5)-I(-1,7)-\tilde{C}_{i} I(-1,5)\right]\right. \\
& \left.+X_{e} \mu_{e}^{5 / 2}\left[E(1,7)+\tilde{C}_{e} E(1,5)-E(-1,7)-\tilde{C}_{e} E(-1,5)\right]\right\}, \\
& \chi_{x y}^{1 R}=\frac{A}{15(2 \pi)^{1 / 2}}\left\{\frac { X _ { i } \mu _ { i } ^ { 5 / 2 } } { Y _ { i } ^ { 2 } } \left\{I(2,9)+\tilde{C}_{i} I(2,7)-I(-2,9)-\tilde{C}_{i} I(-2,7)\right.\right. \\
& \left.-2\left[I(1,9)+\tilde{C}_{i} I(1,7)+2 I(-1,9)+\tilde{C}_{i} I(-1,7)\right]\right\} \\
& +\frac{X_{e} \mu_{e}^{5 / 2}}{Y_{e}^{2}}\left\{E(2,9)+\tilde{C}_{e} E(2,7)-E(-2,9)-\tilde{C}_{e} E(-2,7)\right. \\
& \left.\left.-2\left[E(1,9)+\tilde{C}_{e} E(1,7)+2 E(-1,9)+\tilde{C}_{e} E(-1,7)\right]\right\}\right\}, \\
& \chi_{x y}^{0 I}=\frac{1}{3(2 \pi)^{1 / 2}}\left\{X_{i} \mu_{i}^{5 / 2}\left[\left(1+Y_{i}\right) I(1,6)-\left(1-Y_{i}\right) I(-1,6)\right]\right. \\
& \left.+X_{e} \mu_{e}^{5 / 2}\left[\left(1+Y_{e}\right) E(1,6)-\left(1-Y_{e}\right) E(-1,6)-\frac{2 Y_{e}}{1-Y_{e}^{2}} i_{4}\right]\right\},
\end{aligned}
$$




$$
\begin{aligned}
\chi_{x y}^{1 I}= & \frac{1}{15(2 \pi)^{1 / 2}}\left\{\frac { X _ { i } \mu _ { i } ^ { 5 / 2 } } { Y _ { i } ^ { 2 } } \left[\left(1+2 Y_{i}\right) I(2,8)-\left(1-2 Y_{i}\right) I(-2,8)\right.\right. \\
& \left.-2\left(1+Y_{i}\right) I(1,8)+2\left(1-Y_{i}\right) I(-1,8)\right] \\
& +\frac{X_{e} \mu_{e}^{5 / 2}}{Y_{e}^{2}}\left[\left(1+2 Y_{e}\right) E(2,8)-\left(1-2 Y_{e}\right) E(-2,8)\right. \\
& -2\left(1+Y_{e}\right) E(1,8)+2\left(1-Y_{e}\right) E(-1,8) \\
& \left.\left.-\frac{4 Y_{e}}{1-4 Y_{e}^{2}} i_{6}+\frac{4 Y_{e}}{1-Y_{e}^{2}} i_{6}\right]\right\} .
\end{aligned}
$$

\section{Appendix D}

The full expressions for the $F_{i j}^{R}$ and $F_{i j}^{I}$ are as follows:

with

$$
\begin{aligned}
& F_{i j}^{R}=u_{i}^{R} s_{j}^{R}-u_{i}^{I} s_{j}^{I}, \\
& F_{i j}^{I}=u_{i}^{R} s_{j}^{I}+u_{i}^{I} s_{j}^{R},
\end{aligned}
$$

$$
\begin{gathered}
u_{x}^{R}=\frac{\omega_{p i}^{2} \mu_{i}^{3 / 2}}{Y_{i}}\left[\left(1+Y_{i}\right) I(1,5)-\left(1-Y_{i}\right) I(-1,5)\right] \\
+\frac{\omega_{p e}^{2} \mu_{e}^{3 / 2}}{Y_{e}}\left[\left(1+Y_{e}\right) E(1,5)-\left(1-Y_{e}\right) E(-1,5)\right], \\
u_{x}^{I}=-A\left\{\frac{\omega_{p i}^{2} \mu_{i}^{3 / 2}}{Y_{i}}\left[I(1,6)+\tilde{C}_{i} I(1,4)-I(-1,6)-\tilde{C}_{i} I(-1,4)\right]\right. \\
\left.+\frac{\omega_{p e}^{2} \mu_{e}^{3 / 2}}{Y_{e}}\left[E(1,6)+\tilde{C}_{e} E(1,4)-E(-1,6)-\tilde{C}_{e} E(-1,4)\right]\right\}, \\
s_{x}^{R}=\frac{\omega_{p i}^{2} \mu_{i}^{5 / 2}}{Y_{i}}\left\{\left(1+Y_{i}\right)\left[I(1,7)+\tilde{C}_{i} I(1,5)\right]\right. \\
\left.-\left(1-Y_{i}\right)\left[I(-1,7)+\tilde{C}_{i} I(-1,5)\right]\right\} \\
+\frac{\omega_{p e}^{2} \mu_{e}^{5 / 2}}{Y_{e}}\left\{\left(1+Y_{e}\right)\left[E(1,7)+\tilde{C}_{e} E(1,5)\right]\right. \\
\left.-\left(1-Y_{e}\right)\left[E(-1,7)+\tilde{C}_{e} E(-1,5)\right]\right\}, \\
s_{x}^{I}=-A\left\{\frac { \omega _ { p i } ^ { 2 } \mu _ { i } ^ { 5 / 2 } } { Y _ { i } } \left[I(1,8)+2 \tilde{C}_{i} I(1,6)+\tilde{C}_{i}^{2} I(1,4)\right.\right. \\
\left.-I(-1,8)-2 \tilde{C}_{i} I(-1,6)-\tilde{C}_{i}^{2} I(-1,4)\right] \\
+\frac{\omega_{p e}^{2} \mu_{e}^{5 / 2}}{Y_{e}}\left[E(1,8)+2 \tilde{C}_{e} E(1,6)+\tilde{C}_{e}^{2} E(1,4)\right. \\
\left.\left.-E(-1,8)-2 \tilde{C}_{e} E(-1,6)-\tilde{C}_{e}^{2} E(-1,4)\right]\right\}, \\
u_{y}^{R}=\frac{\omega_{p i}^{2} \mu_{i}^{3 / 2}}{Y_{i}}\left[2 I(0,5)-\left(1+Y_{i}\right) I(1,5)-\left(1-Y_{i}\right) I(-1,5)\right] \\
{\left[2 E(0,5)-\left(1+Y_{e}\right) E(1,5)-\left(1-Y_{e}\right) E(-1,5)\right],}
\end{gathered}
$$




$$
\begin{aligned}
& u_{y}^{I}=-A\left\{\frac { \omega _ { p i } ^ { 2 } \mu _ { i } ^ { 3 / 2 } } { Y _ { i } } \left\{2\left[I(0,6)+\tilde{C}_{i} I(0,4)\right]-I(1,6)-\tilde{C}_{i} I(1,4)\right.\right. \\
&\left.-I(-1,6)-\tilde{C}_{i} I(-1,4)\right\} \\
&+\frac{\omega_{p e}^{2} \mu_{e}^{3 / 2}}{Y_{e}}\left\{2\left[E(0,6)+\tilde{C}_{e} E(0,4)\right]-E(1,6)-\tilde{C}_{e} E(1,4)\right. \\
&\left.\left.-E(-1,6)-\tilde{C}_{e} E(-1,4)\right\}\right\} \\
& s_{y}^{R}=\frac{\omega_{p i}^{2} \mu_{i}^{5 / 2}}{Y_{i}}\left\{-2\left[I(0,7)+\tilde{C}_{i} I(0,5)\right]\right. \\
&\left.+\left(1+Y_{i}\right)\left[I(1,7)+\tilde{C}_{i} I(1,5)\right]+\left(1-Y_{i}\right)\left[I(-1,7)+\tilde{C}_{i} I(-1,5)\right]\right\} \\
&+\frac{\omega_{p e}^{2} \mu_{e}^{5 / 2}}{Y_{e}}\left\{-2\left[E(0,7)+\tilde{C}_{e} E(0,5)\right]\right. \\
&\left.+\left(1+Y_{e}\right)\left[E(1,7)+\tilde{C}_{e} E(1,5)\right]+\left(1-Y_{e}\right)\left[E(-1,7)+\tilde{C}_{e} E(-1,5)\right]\right\} \\
& s_{y}^{I}=-A\left\{\frac { \omega _ { p i } ^ { 2 } \mu _ { i } ^ { 5 / 2 } } { Y _ { i } } \left\{-2\left[I(0,8)+2 \tilde{C}_{i} I(0,6)+\tilde{C}_{i}^{2} I(0,4)\right]\right.\right. \\
&+I(1,8)+2 \tilde{C}_{i} I(1,6)+\tilde{C}_{i}^{2} I(1,4) \\
&\left.+I(-1,8)+2 \tilde{C}_{i} I(-1,6)+\tilde{C}_{i}^{2} I(-1,4)\right\} \\
&+\frac{\omega_{p e}^{2} \mu_{e}^{5 / 2}}{Y_{e}}\left\{-2\left[E(0,8)+2 \tilde{C}_{e} E(0,6)+\tilde{C}_{e}^{2} E(0,4)\right]\right. \\
&+E(1,8)+2 \tilde{C}_{e} E(1,6)+\tilde{C}_{e}^{2} E(1,4) \\
&\left.\left.+E(-1,8)+2 \tilde{C}_{e} E(-1,6)+\tilde{C}_{e}^{2} E(-1,4)\right\}\right\}
\end{aligned}
$$

\section{References}

Abramowitz, M. and Stegun, I. A. 1970 Handbook of Mathematical Functions. Dover, New York.

Chang, J.S. and Spariosu, K. 1993 Dust particle charging characteristics under a collisionless magneto-plasma. J. Phys. Soc. Japan 62, 97.

Cramer, N. F. and Vladimirov, S. V. 1998 The resonance absorption of wave energy in a dusty plasma. Physica Scripta T45, 213.

de Juli, M. C. and Schneider, R. S. 1998 The dielectric tensor for dusty magnetized plasmas with variable charge on dust particles. J. Plasma Phys. 60, 243.

Hasegawa, A. 1976 Kinetic processes in plasma heating by resonant mode conversion of Alfvén wave. Phys. Fluids 19, 1924.

Prasad, P. V. S. R. 1998 Acoustic modes in a dusty plasma. Phys. Lett. 239A, 378.

Rao, N. N. 1995 Magnetoacoustic modes in a magnetized dusty plasma. J. Plasma Phys. 53, 317.

Spitzer, L. J. 1978 Physical Processes in the Interstellar Medium. Wiley, New York.

Tsytovich, V. N. 1997 Dust plasma crystals, drops and clouds. Soviet Phys. Usp. 40, 53.

Vladimirov, S. V. 1994 Propagation of waves in dusty plasmas with variable charges on dust particles. Phys. Plasmas 1, 2762. 